\begin{tabular}{|c|c|c|c|c|c|}
\hline MUNIBE Antropologia-Arkeologia & $n^{\circ} 66$ & $135-145$ & DONOSTIA & 2015 & ISSN 1132-2217 • eISSN 2172-4555 \\
\hline
\end{tabular}

\title{
Las inhumaciones del Neolítico antiguo de Ca I’Estrada (Canovelles, Barcelona)
}

\author{
The ancient Neolithic burials of Ca l'Estrada (Canovelles, Barcelona)
}

PALABRAS CLAVES: Neolítico Antiguo, noreste peninsular, prácticas funerarias, estudio antropológico, Ca l'Estrada. GAKO-HITZAK: Neolito Zaharra, penintsulako ipar-ekialdea, hileta-ohiturak, ikerketa antropologikoa, Ca l'Estrada. KEY WORDS: Ancient Neolithic, Peninsular Northeast, funerary practices, Anthropological study, Ca l'Estrada.

\section{Maria EULÀLIA SUBIRÀ(1), Pablo MARTíNEZ(2), Abel FORTÓ(3) Vanessa MUÑOZ(4), David ORTEGA(5) \& Juan F. GIBAJA(5)}

\section{RESUMEN}

Actualmente, en el noreste de la Península Ibérica, se conocen muy pocos restos humanos pertenecientes a las primeras comunidades agricultoras y pastoriles de inicios del neolítico. Buena parte de las evidencias que tenemos provienen de contextos arqueológicos excavados con anterioridad a los años 80 , lo que supone que no se dispone del mismo acerbo de análisis que se realizan en la actualidad. La excavación realizada en Ca l'Estrada viene a rellenar en parte ese vacío aportando no sólo dos nuevos enterramientos, sino también toda una serie de análisis tanto del continente como del contenido de las sepulturas. Su estudio nos ha permitido conocer biológicamente a dos de los sujetos protagonistas de aquella su historia, cómo fueron inhumados y qué objetos les acompañaban. Estamos, sin duda, ante una de las primeras evidencias funerarias de lo que ha venido a denominarse como "la Cultura de los Sepulcros de fosa".

\section{LABURPENA}

Gaur egun Iberiar Penintsularen Ipar-Ekialdean bizi izan ziren lehen talde nekazari eta artzainen giza aztarna gutxi ezagutzen dira. Ezagutzen diren ebidentzia gehienak 1980ko hamarkada baino lehenagoko indusketa arkeologikoei dagokie, eta horren ondorioz, egun egiten diren hainbat analisiren falta dago testuinguru hauetan. Zentzu honetan Ca l'Estradan egindako indusketak hutsune hau bete egiten du, hilobi berri bi ezagutarazteaz gain, bai honen egituraren zein bere edukiaren gain egin diren azterketa guztiek aurkezten duten datuengatik. Ikerketa honek bi gizabanakoren, lurperatzearen testuingurua zein agertzen diren materialei buruzko informazioa eskaintzen du. Argi dago "la Cultura de los Sepulcros de fosa" deritzonaren lehen aztarnetariko baten inguruan ari garela lan honetan.

\section{ABSTRACT}

At present there are few human remains from early agricultural and pastoralists communities of the early Neolithic in the northeast of the Iberian Peninsula Most of the evidence comes from archaeological contexts excavated prior to the 80's, then recent analytical are absent. The excavation in Ca l'Estrada partly fills this gap providing not only two new burials, but also a whole set of analyzes of the graves Their study has allowed us biologically meet two of the subjects protagonists of that history, how they were buried and what objects accompanied them. We are certainly at an early funerary evidence of what has come to be known as "Culture of pit graves."

There are two burials; The first one comes from a cuvette of $70-80 \mathrm{~cm}$ wide and $60-70 \mathrm{~cm}$ deep. A woman $25-35$ years old very well represented was depositated on right lateral decubitus with flexed limbs and his body was enveloped into a bundle or shroud. Under the pelvis a sheet of white flint good was found. This blade was used to cut plants near the ground or on it before putting it as dowry.

It could not define the structure of the second burial. The remains correspond a part of a child of 5-6 yearsold, possibly a male.

The study allows start to know biologicaly the human group that lived the zone. Likewise the detailed study of his grave has allowed to propose that it was wrapped in a shroud, deposited gathered and with the presence of a sheet of silex, attends that it might start to justify a ritual that will become more frequent in the Middle Neolithic.

(1) GRAPAC.Unitat d'Antropologia Biològica. Departament de biologia animal, de biologia vegetal i d'ecologia. U.A.B.08103 Bellaterra Eulalia.Subira@uab.cat

(2) Arqueòleg. Collectiu per a la Investigació de la Prehistòria i Arqueologia de Garraf. Membre del Seminari d'Estudis i Recerca Prehistòrica (SERP), Universitat de Barcelona. pablomartrod@gmail.com

(3) Unitat de Recerca Històrica. Patrimoni Cultural d'Andorra. Carretera de Bixessarri s/n, Aixovall. AD 600, Sant Julià. Andorra. abel_forto@govern.ad (4) Fragments ®. Plaça Can Gassol núm 18, 08301 Mataró. info@fragments.es

(5)IMF-CSIC, C/Egipcíaques 15, Barcelona, E-08001, España. ortega@imf.csic.es; jfgibaja@imf.csic.es 


\section{1.- INTRODUCCIÓN}

\subsection{Las sepulturas de Ca l'Estrada en el contexto del neolítico antiguo del noreste de la Península Ibérica}

Son pocas las evidencias que se tienen de contextos funerarios relacionados con los primeros grupos de agricultores y pastores del neolítico. Este vacio va llenándose gracias a los descubrimientos realizados en los últimos años y a las dataciones sobre restos humanos que han ido efectuándose.

A este respecto, en la plaza Vila de Madrid, de la ciudad de Barcelona, se localizó la inhumación de un individuo juvenil que estaba acompañado de unas pocas lascas de sílex y jaspe. La datación realizada sobre los restos humanos de dicho individuo ofreció la fecha neolítica más antigua de un contexto funerario: $6440 \pm 40 \mathrm{BP}$ (5535-5460 Cal BC at 2 sigmas) (POU et al., 2010).

Casi contemporáneas son las inhumaciones documentadas en la capa 18 de la cova de Can Sadurní. Allí se hallaron varios enterramientos próximos a magníficos vasos de cerámica decorados con impresión cardial y rellenos con numerosas semillas carbonizadas. Precisamente, las dataciones realizadas en dos de esas semillas ofrecieron los siguientes resultados: $6405 \pm 55$ BP (5476$5309 \mathrm{cal} \mathrm{BC})$ y $6421 \pm 34$ BP (5473-5329 cal BC) (BLASCO et al., 2005). Asimismo, en la capa 10 de esta misma cueva se documentaron varias inhumaciones cronológicamente más recientes pertenecientes al neolítico antiguo postcardial (VILLALBA et al., 2004, 2006).

A partir aproximadamente de la segunda mitad del $\mathrm{V}$ milenio cal BC, aumenta progresivamente el número de sepulturas y su variedad en relación a su ubicación y al tipo de estructura funeraria. Si las cuevas siguen reutilizándose como espacios funerarios colectivos, como es el caso de la Cova de l'Avellaner, la Cova de les Grioteres o la Cova del Pasteral (TEN, 1980; BOSCH \& TARRÚS, 1991; CASTANY, 1992), paralelamente parecen construirse las primeras construcciones megalíticas en yacimientos como Rajols, Font de la Vena y Padró (MOLIST et al., 1987). Las dataciones realizadas sobre muestras de carbón de Padró II ofrecieron los datos siguientes: $5870 \pm 100 \mathrm{BP}(4986-4501 \mathrm{cal} \mathrm{BC})$ y $5770 \pm 80 \mathrm{BP}(4801$ - 4451 cal BC). Pensamos que tales fechas deberían revisarse, en tanto que no se están fechando a los inhumados directamente y las muestras de carbón pueden estar dando fechas más antiguas si tenemos en cuenta el efecto de la "madera vieja".

Por su parte, en la llanura de Barcelona se han descubierto desde los años 90 un conjunto de yacimientos, algunos de los cuales presentan estructuras funerarias y dataciones similares a las de Ca l'Estrada. Si el más conocido por su extensión, amplia secuencia histórica y la existencia de una de las necrópolis neolíticas más antiguas con 24 enterramientos, es Sant Pau del Camp (GRANADOS et al., 1993; GIBAJA, 2003; MOLIST et al., 2008), a pocos metros se excavó en 2008-2009 el yacimiento del Carrer Reina Amàlia. En dicho yacimiento se hallaron varias sepulturas, una de las cuales se localizó en el interior de una estructura de hábitat.

Las fechas de C14 realizadas en Sant Pau del Camp dieron resultados negativos y únicamente puede ser una referencia, con ciertas precauciones, la datación obtenida en la sepultura 18: 5160 \pm 130 BP (4250-3700 cal $\mathrm{BC}$ ). En cambio, los datos cronométricos de las sepulturas del Carrer Reina Amàlia son más fiables e interesantes ya que ofrecieron: $5750 \pm 40 \mathrm{BP}(4690-4460 \mathrm{cal} \mathrm{BC})$ para uno de los enterramientos infantiles (UF1) hallado en el interior de la cabaña, y 5520 $\pm 40 \mathrm{BP}(4530-4360 \mathrm{cal}$ $\mathrm{BC}$ ) para el enterramiento de un adulto (UF3) documentado en el nivel de abandono de la UE15 (FORTÓ et al., 2006; GONZÁLEZ et al., 2011).

Si bien es posible que otras inhumaciones también pertenezcan al horizonte cronológico del neolítico antiguo, caso de los enterramientos de las Bocas del Ebro, por el momento no tenemos dataciones absolutas que confirmen dicha aseveración.

A finales del $\mathrm{V}$ milenio cal BC, y especialmente a lo largo del IV, las poblaciones neolíticas empiezan a enterrar a parte de sus muertos de manera sistemática, ya sea en sepulturas aisladas, en agrupaciones en cueva o en necrópolis con tumbas individualizadas y visibles en el mismo espacio. Tal es la importancia de las estructuras funerarias de este momento, que ya a inicios del s.XX el profesor J. Bosch Gimpera (1913-14) las incluyó en su tercera civilización de Catalunya, que denominó "Cultura de los Sepulcros no Megalíticos" y al que con posterioridad se conoció como "La Cultura de los Sepulcros de Fosa". Aunque hoy es difícil calcular cuántos enterramientos pertenecen a esta "Cultura", pues muchos fueron destruídos antiguamente como consecuencia de las innumerables actividades agrícolas e industriales, probablemente se superen los 650

En este contexto, la datación de la inhumación de $\mathrm{Ca}$ l'Estrada es enormemente relevante por su antigüedad y por la escasez de fechas que hay sobre sepulturas de esa cronología.

\subsection{Ca l'Estrada}

El yacimiento de Ca l'Estrada se localizó durante los trabajos de prospección arqueológica de urgencia realizados entre el 2003 y el 2005 en la Ronda Norte de Granollers (Canovelles, Barcelona), en el lado este del curso actual del Torrente d'en Fangues (Figura 1). El hecho de que este torrente, afluente del río Congost, atravesara en épocas pasadas esta llanura, una vez superado un tramo donde el cauce se encontraba muy encajonado, provocó un cambio continuo en el relieve de esta zona como consecuencia de los numerosos episodios de desbordamiento e inundación que había sufrido. No obstante, este paraje debió ser enormemente atractivo para las comunidades agricultoras y pastoras del neolítico, pues había terrenos bien regados, aptos para las prácticas subsistenciales. 


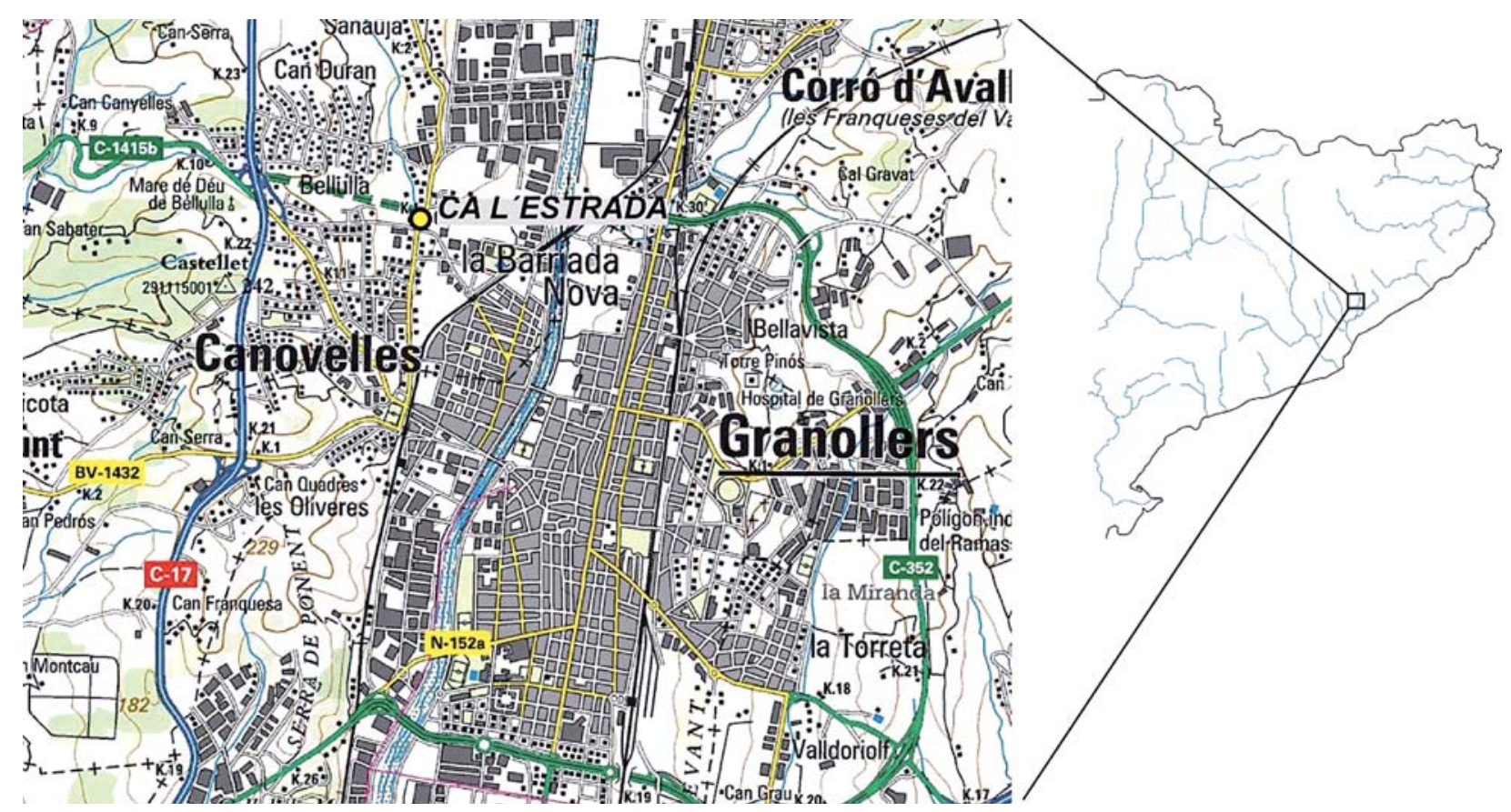

Fig. 1. Mapa de Catalunya con la situación del yacimiento de Ca l'Estrada. / Ca l'Estrada location in catalonia.

La excavación de Ca l'Estrada permitió documentar diversas ocupaciones humanas sucesivas pero intermitentes en el tiempo. Se inician en la prehistoria, con elementos característicos del neolítico antiguo y del neolítico final-calcolítico, seguidas de ocupaciones del final del periodo Ibérico, del mundo tardoantiguo y de época medieval. Una vez agotada la estratigrafía perteneciente a las cronologías históricas, se localizaron diferentes estructuras y materiales correspondientes al neolítico final (FORTÓ et al., 2005, 2006, 2008), así como dos enterramientos neolíticos. Aunque el yacimiento ha sido motivo de varias publicaciones, ninguna por el momento se había centrado en tales sepulturas. Su importancia es tan relevante que, sin duda, merecían artículo monográfico como el que se presenta en estas páginas.

\section{2.- METODOLOGÍA}

\subsection{Análisis Antropológico}

En el campo se documentó la posición de cada una de las piezas óseas y sus relaciones articulares, así como su vinculación con el espacio del enterramiento, tal y como sugiere Duday (et al., 1990; 2009) con el fin de establecer los distintos procesos tafonómicos ocurridos y poder reconstruir de esa manera el proceso de enterramiento de los cadáveres.

Se trata de dos individuos sobre los que se ha calculado, en primer lugar, los índices de preservación. Para ello se han empleado los criterios apuntados por Walker y otros (1988) y modificadas por Safont y otros (1999). Su análisis aporta información de las partes esqueléticas preservadas (IP1, huesos largos; IP2 huesos largos y cin- tura espacular y pélvica; y IP3, que incluye además diversas partes craneales), pero no así el grado de conservación de los distintos elementos óseos.

Para el diagnóstico del sexo de los individuos, se ha analizado la morfología de la pelvis (FÉREMBACH et al., 1980; KROGMAN E ISCAN, 1986; SCHTKOWSKI, 1993) y del cráneo (FÉREMBACH et al., 1980).

La edad de los individuos se ha calculado en función del desarrollo dental (CRÉTOT, 1978; UBELAKER, 1989), de la superficie auricular de la pelvis (MEINDL Y LOVEJOY, 1985) y de las suturas craneales, por este orden (LOVEJOY et al., 1985; MASSET, 1982).

El análisis métrico y la caracterización tipológica se ha basado en los criterios establecidos por Martin y Saller (1957), y Olivier (1960).

\subsection{Análisis de la industria lítica}

Las materias primas líticas (sílex) se han clasificado atendiendo a sus caracteres macroscópicos principales (textura, color, opacidad, etc.), usando para ello una lupa binocular. Para determinar su origen geológico y eventual área de procedencia, se han comparado los artefactos arqueológicos con las muestras y descripciones de rocas silíceas de la región NE peninsular de las colecciones Litocat, en la Institución Milà i Fontanals, en Barcelona (TERRADAS et al., 2012). Para el análisis tecnológico de las producciones laminares se ha recurrido a la descripción de los atributos y estigmas de talla característicos de las distintas técnicas de lascado referidas en Inizian y otros (1995) y Palomo (2012). 
Para el estudio funcional de la pieza de sílex documentada en el interior de la tumba SF-501 hemos empleado una lupa binocular Leica MZ16A, que abarca entre 10-90 aumentos, y un microscopio metalográfico Olympus $\mathrm{BH} 2$, cuyos aumentos van desde 50X a 400X. Antes de iniciar la limpieza y su posterior análisis, hemos efectuado una primera observación bajo lupa binocular con el objetivo de detectar y registrar todos aquellos posibles residuos orgánicos e inorgánicos que pudieran estar aún adheridos a la superficie de la pieza. Posteriormente, hemos realizado una limpieza simplemente con agua y jabón. No ha sido necesario el uso de soluciones ácidas para eliminar concreciones calcáreas pues no existían.

\section{3.- RESULTADOS}

\subsection{Sepulturas de Ca l'Estrada: estructura, modo de enterramiento y cultura material}

La información obtenida del estudio de las dos sepulturas documentadas quedan resumidas en las siguientes líneas:

Sepultura SF-501: Hallada en la rasa número 18, a unos 2,10 metros de profundidad (Figura 2), los restos humanos encontrados en el interior de esta estructura funeraria corresponden a una inhumación primaria de un individuo adulto, en conexión anatómica y dispuesto en posición decúbito lateral derecho (Figura 3 y 4). Para delimitar las dimensiones de la fosa o el recorte en el que estaba dispuesto el enterramiento, se aprovechó el rebaje de la máquina que lateralmente había llegado a una cota inferior y había dejado la inhumación vista en el perfil. La estructura corresponde a una cubeta de $70-80 \mathrm{~cm}$ de ancho y 60-70 cm de profundidad.

A continuación se delimitó una área de $20 \mathrm{~m}^{2}$ a su alrededor y se hicieron rebajes controlados con el fin de conocer y documentar la posible existencia de otras estructuras.

La datación de C14 (AMS) sobre uno de los huesos

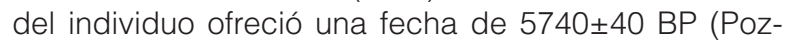
10391), cuya calibración a dos sigmas, mediante el programa OxCal 4.1.5 (BRONK, 2010), supone un intervalo cronológico entre 4694-4491 cal. BC (FORTÓ et al., 2006). Ello sitúa a esta inhumación en un periodo de transición entre lo que ha venido a catalogarse como el Neolítico Antiguo Epicardial y el Neolítico Antiguo Postcardial (BLASCO et al., 2005).

En el interior de la sepultura, acompañando al individuo, se halló una lámina de sílex blanco que estaba dispuesta bajo su pelvis. Se trata de un tipo de sílex cuya procedencia es ajena al contexto geológico local de la comarca del Vallés, por lo que evidentemente fue importado. Sin más material del mismo tipo, y al ser imposible emplear técnicas petrográficas, su identificación y atribución a una formación geológica concreta resulta irrealizable. No obstante, cabe decir que pueden hallarse rocas de aspecto parecido en formaciones continentales evaporíticas (yesos) de edad eocena en el márgen cata- lánide de la Cuenca del Ebro, en las comarcas del Priorat y la Cuenca de Barberà (ORTí, 1990), así como en el Prepirineo de Lleida, en la formación Tremp, de edad daniense, que incluye facies sedimentarias continentales afines a aquellas (ROSELL et al., 2001).

Desde el punto de vista técnico se trata de un producto laminar de buena regularidad que mide $75 \times 21 \times 4$ $\mathrm{mm}$. Presenta en la cara dorsal 4 negativos de extraciones de idéntica polaridad a la del soporte y, en el extremo proximal, un contrabulbo muy marcado con un talón diedro y profundo (6 $\mathrm{mm}$ de espesor). Todo este conjunto de carácteres apuntarían al uso de la percusión indirecta como técnica de talla probablemente empleada.

El análisis traceológico ha permitido observar que esta lámina fue utilizada previamente a dejarla en la tumba para cortar plantas cerca del suelo o sobre éste. Tal actividad suele estar relacionada con la separación de la espiga/raíces del tallo o con el corte de tales tallos en una medidas determinadas para hacer cestería, techumbres, etc (CLEMENTE Y GIBAJA, 1998; GIBAJA, 2003) (Figura 5).

Fuera de las inhumaciones se documentaron algunos fragmentos de cerámica atribuíbles al neolítico antiguo epicardial. Más allá de que en otros muchos yacimientos de Cataluña haya cerámicas similares, cabe destacar el del Pla del Serrador, situado a 1500 metros respecto a Ca l'Estrada, donde se localizó un silo con material claramente Epicardial datado en 5810 440 BP (MUÑOZ, 2005), cuya calibración corresponde al intervalo $4778-4548$ cal BC. Lo que supone que ambos contextos serían contemporáneos.

Sepultura SF-502: Al continuar con la prospección en la zona circundante alrededor de la anterior sepultura, se localizó un segundo enterramiento a un metro escaso de distancia y a la misma profundidad. Los trabajos de rebaje con maquinaria, dada la profundidad de la primera, afectaron a la estructura funeraria, destruyéndola en parte e imposibilitando su descripción. En este caso, se recuperaron los restos de un individuo infantil que se encontraba en un pésimo estado de conservación. In situ solamente se localizaron el antebrazo izquierdo y parte de la caja torácica (Figura 6). El resto de fragmentos se hallaron durante el proceso de cribado de la tierra extraída.

\subsection{Sepulturas de Ca l'Estrada: estudio antro- pológico}

SF-501: El individuo apareció en decúbito lateral derecho con el torso en vista posterior. Las piernas estaban totalmente plegadas sobre si mismas, así como el cuerpo flexionado. Las extremidades superiores también se encontraban flexionadas, en un ángulo de $45^{\circ}$ la izquierda y cercano a los $90^{\circ}$ la derecha, y la cabeza orientada $240^{\circ}$ al oeste y los pies $60^{\circ}$ al este, con la cara mirando hacia el este (Figura 4). Las conexiones de las articulaciones eran, en general, estrechas, lo que indica que el cadáver se colmató de tierra después de su depósito. 
PLÀNOL GENERAL

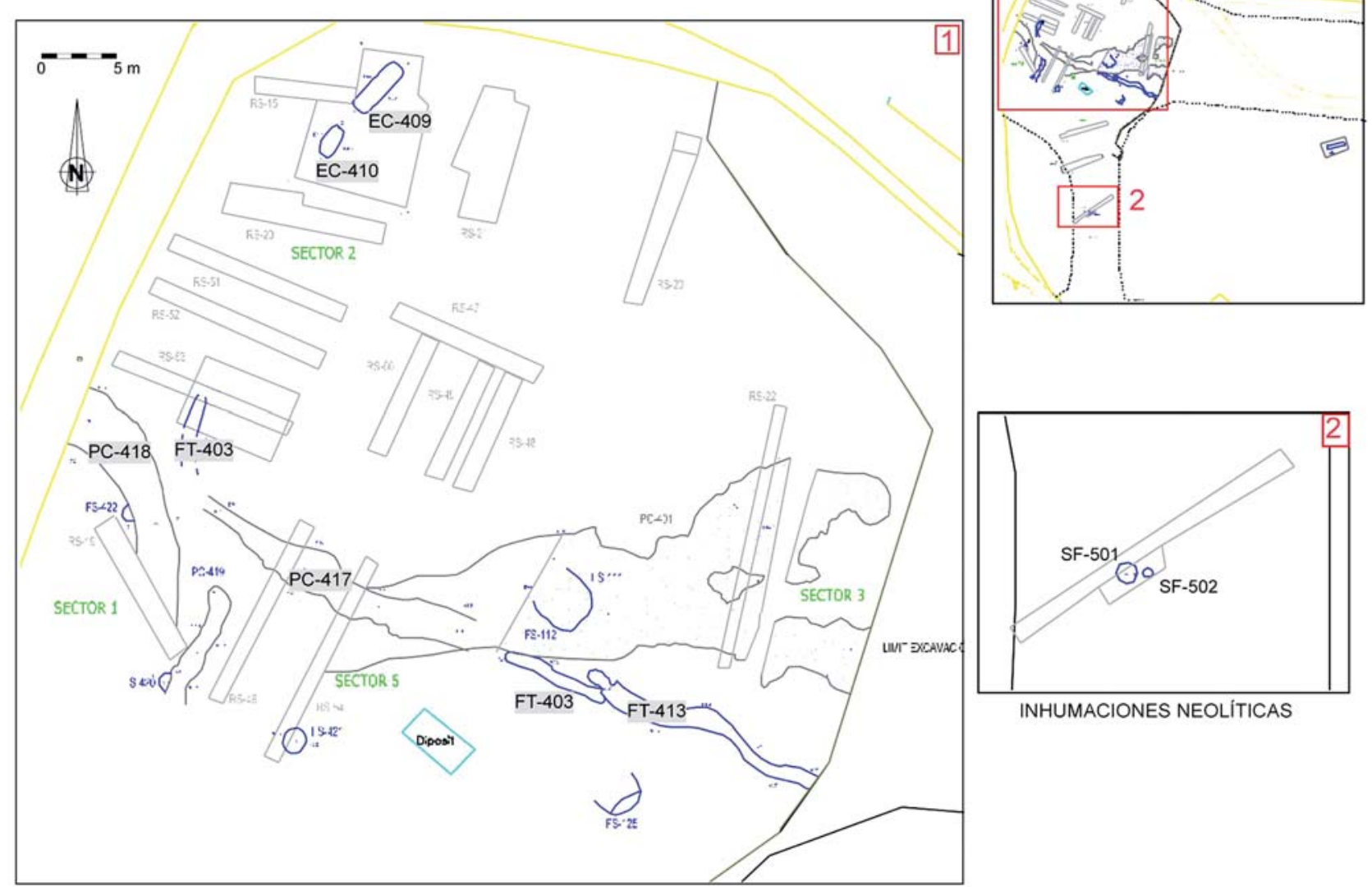

Fig. 2. Mapa general del yacimiento con la situación de las inhumaciones. / Overview of Ca l'Estrada with location of the burials.

La extremidad superior izquierda abrazaba las rodillas recogiendo las piernas; la posición de las extremidades inferiores muy forzada. La espalda del individuo se había desplazado hacia delante, ya que probablemente en inicio estaría en posición totalmente lateral, apoyándose en la pared de la propia estructura. El peso de la tierra que colmató la estructura, a la vez que la propia descomposición del individuo, causaron la basculación del cuerpo hacia delante, quedando en la posición dorsal con la que ha sido encontrado. Esta rotación pudo observarse en la posición y en los pequeños movimientos de algunos huesos: la situación dorsal del húmero y la laxitud con el omoplato; la vista posterior del coxal izquierdo que se articula laxamente con el fémur (que se observa en vista lateral al igual que la pierna izquierda); la disposición plana de la escápula izquierda y vertical de la derecha; y la laxitud de las últimas vértebras lumbares.

Asimismo, en la columna vertebral se apreciaba cierta sinuosidad en las vértebras resiguiendo sus apófisis espinosas. Existía cierta laxitud entre el axis, el atlas y el cráneo, observándose este último en norma lateral. El estudio detallado de los leves desplazamientos de las piezas óseas, ya sea de la columna vertebral como de otras articulaciones, permite conocer algún dato de cómo se pro- dujo el depósito del cadáver y su posterior descomposición. Los pequeños desplazamientos a nivel lumbar y del cráneo, así como las diversas dislocaciones de alguna articulación, pueden explicarse por haber estado el cadáver envuelto en una mortaja o fardo. El individuo así dispuesto fue depositado en la tumba y cubierto de tierra, provocando que el cuerpo se abalanzara hacia delante. Esta disposición se diferencia claramente del tipo de depósito que se describirá para el neolítico medio en los llamados sepulcros de fosa, donde los individuos no se depositan tan recogidos, sino que, en su mayoría, se encuentran dispuestos en decúbito supino con la piernas flexionadas. Ejemplos de ello se encuentran en la necrópolis de Can Gambús-Bòbila Madurell (ALLIESE et al., 2014; BRAVO et al., 2015).

El tipo de depósito se asemeja más a los enterramientos mesolíticos del levante peninsular como el de La Corona (FERNÁNDEZ et al., 2012) o El Collado (GIBAJA et al., 2015) en los que los muertos se colocaban dentro de fardos. Sin embargo por su cronología Ca l'Estrada se considera como uno de los enterramientos más antiguos de Catalunya y no sería pues extraño que la tipología del depósito funerario mantuviera todavía las característcas y ciertas tradiciones de los enterramientos anteriores. 


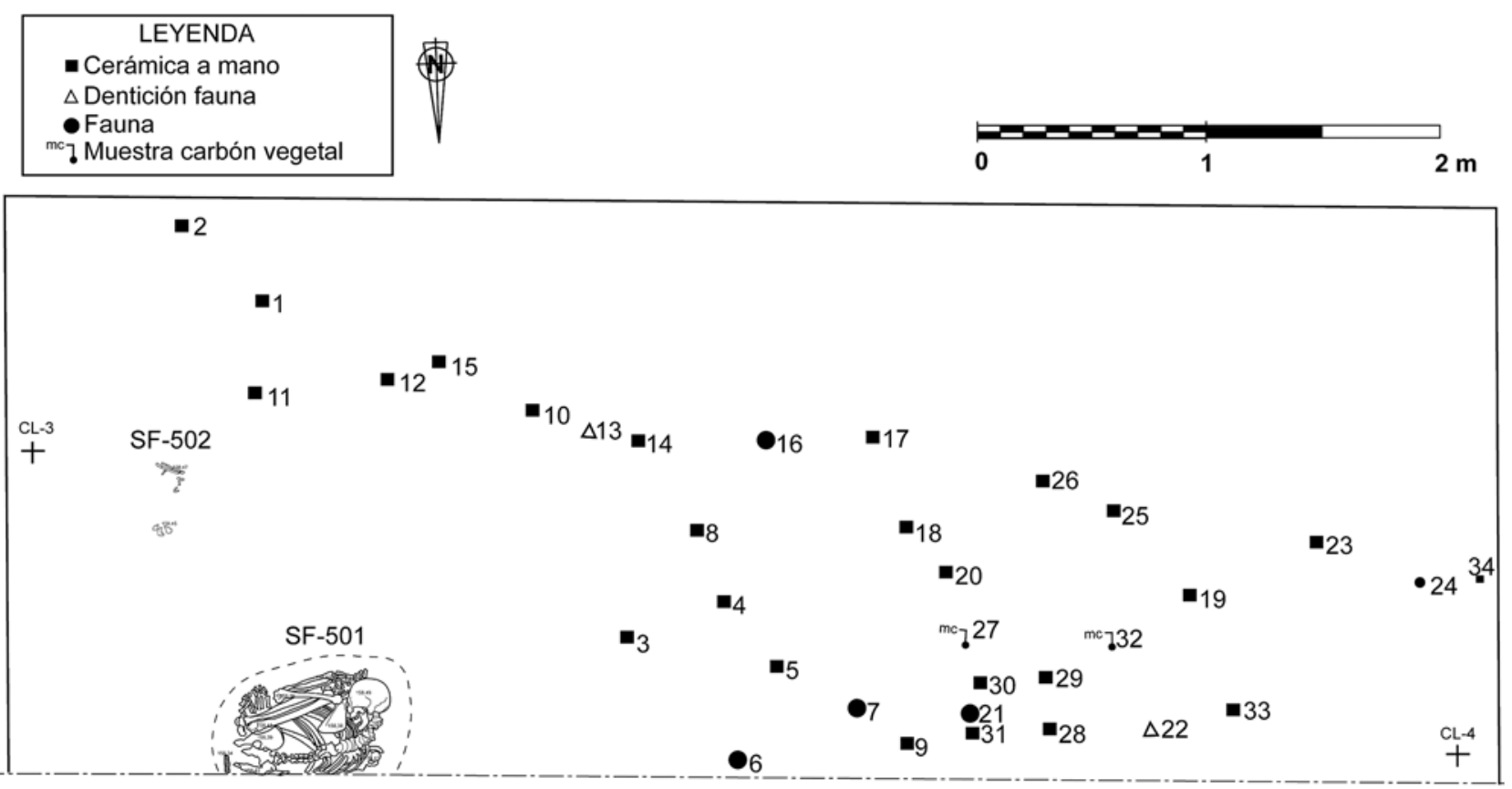

RASA 18

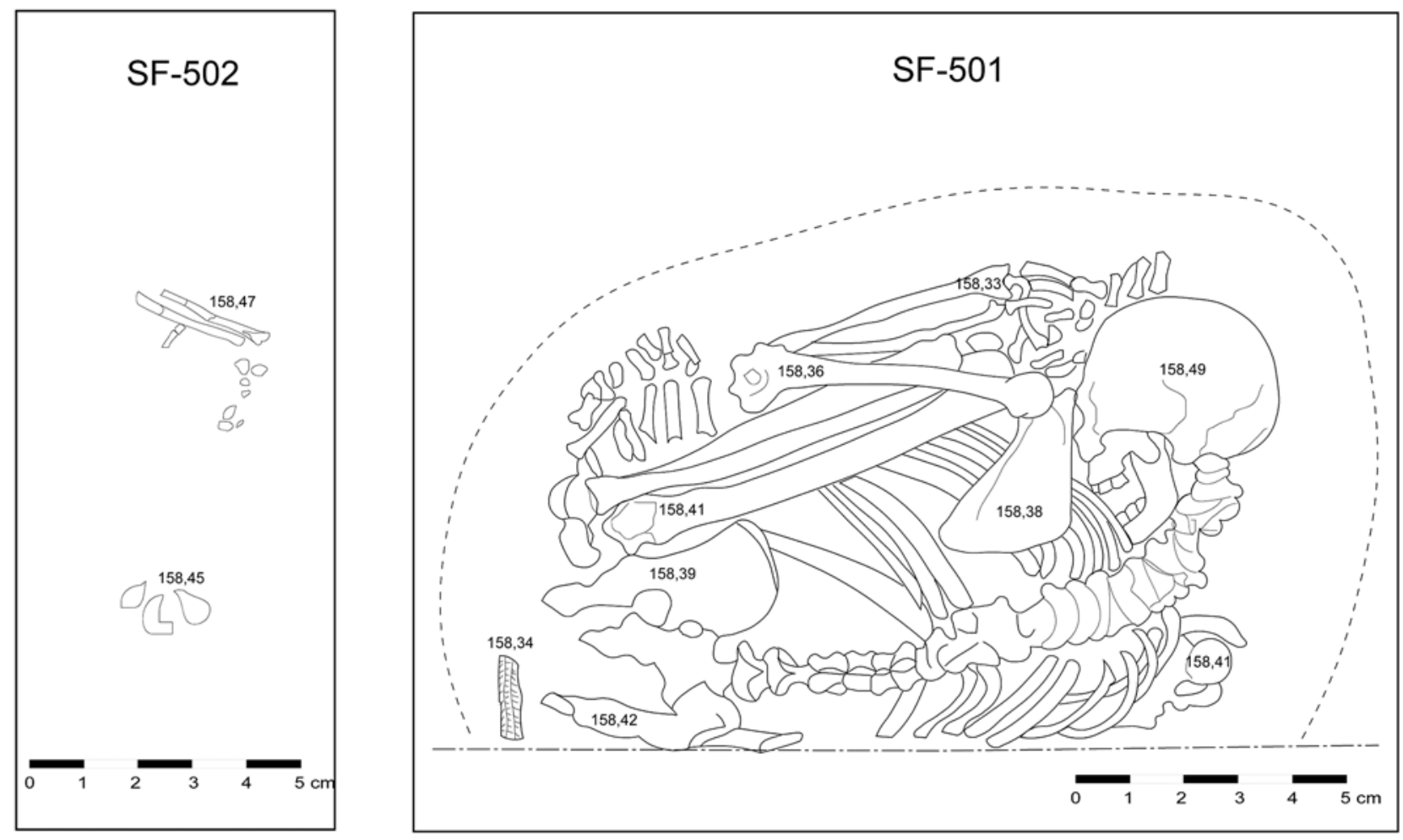

Fig. 3. Planimetría general de las inhumaciones SF-501 y SF-502. / planimetry of the SF-501 and SF-502 burials. 


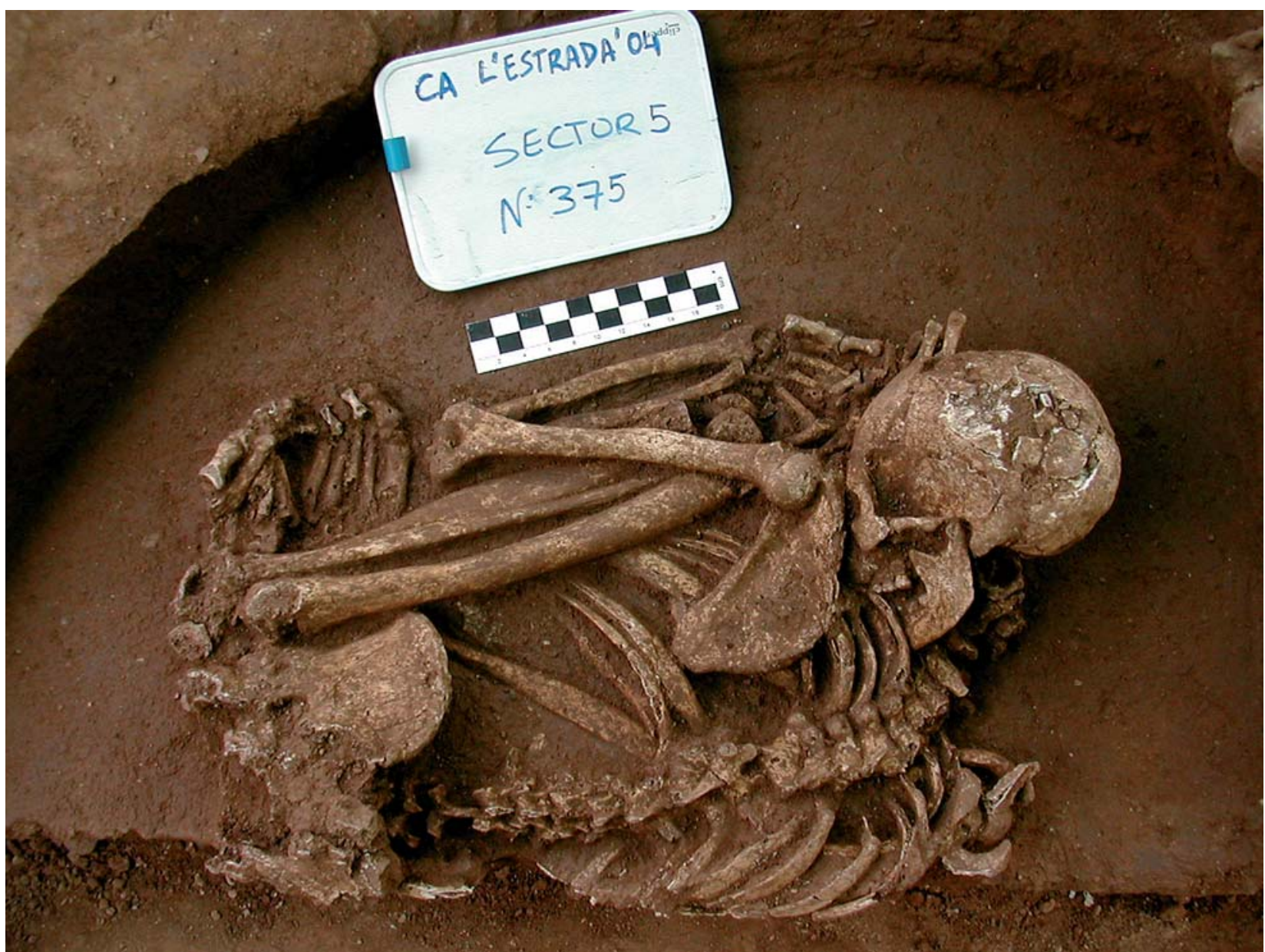

Fig. 4. Fotografía de la inhumación SF-501. / SF-501 skeleton.

Se trata de un individuo muy bien preservado en base a los índices IP $1 \mathrm{P}_{1} \mathrm{IP}_{2}$ y IP $\mathrm{IP}_{3}$ (SAFONT et al., 1999 , WALKER et alii., 1988). En general todas las piezas esqueléticas muestran un buen estado de conservación, tanto las diáfisis como las epífisis. A pesar de ello, algunas regiones del cráneo están deterioradas, por lo que no ha sido posible tomar medidas y establecer su descripción tipológica.

Las características morfológicas de la pelvis (FEREMBACH et al., 1980; KROGMAN \& ISCAN, 1986; LOVEJOY et al., 1985) y del cráneo (FEREMBACH et al., 1980), así como sus rasgos métricos (MARTIN \& SALLER, 1957; OLIVIER, 1960), indican que se trata de un individuo de sexo femenino.

Respecto a la edad, el estudio de la morfología de la superficie auricular (MEINDL \& LOVEJOY, 1985), las suturas craneales (MEINDL \& LOVEJOY, 1985; MASSET, 1982) y el desgaste dental (BROTHWELL, 1987) indican que se trata de un individuo de entre 25 y 35 años.

La comparación de los datos métricos y de los índices de robustez de las extremidades superiores con los de otros individuos femeninos de contextos neolíti- cos, caso de Els Cirerers y Mas d'en Boixos (RUIZ \& SUBIRÀ, 2004; ALFONSO et al., 2000) (tabla I), manifiestan que la mujer de Ca l'Estrada presenta una mayor robustez ósea, observable también en la propia morfología de sus inserciones musculares. Asimismo, dicha robustez se observa más acusada en su lado izquierdo, por lo que probablemente se tratara de una mujer zurda (SUBIRÀ, 2004).

\begin{tabular}{|c|c|c|c|c|c|}
\hline & Els Cirerers & \multicolumn{2}{|c|}{ Mas d'en Boixos } & \multicolumn{2}{|c|}{ Ca l'Estrada } \\
\hline & D & D & 1 & D & 1 \\
\hline Índice diafisario húmero & 100 & - & 75 & 85 & 95,24 \\
\hline Índice de robustez cúbito & & - & & 12,65 & \\
\hline Índice de platolenia cúbito & & 106,66 & & 84,61 & 78,57 \\
\hline Índice de platimería fémur & & - & 66,66 & & 83,87 \\
\hline Índice pilástrico fémur & & - & 128,57 & & - \\
\hline Índice cnémico & & 61,55 & & 63,89 & 56,76 \\
\hline
\end{tabular}

Tabla 1: Índices de robustez de los diferentes huesos largos respecto a individuos femeninos de los yacimientos neolíticos de Els Cirerers y Mas d'en Boixos. / Robustness indices for various long bones of female individuals from the Neolithic sites of Els Cirerers and Mas d'en Boixos. 
La estatura calculada a partir de la única longitud posible, la del cúbito, permite estimar una estatura de unos $166 \mathrm{~cm}$, algo superior a la de los individuos de Mas d'en Boixos (160,03cm), pero parecida al del individuo masculino de Horts de Can Torras (166,34cm) (SUBIRÀ et al., 2004). No obstante, no debe obviarse que son datos aislados y que los cálculos de estatura se han realizado sobre las medidas conservadas en cada caso. En todo caso, y en base a los valores empleados por Campillo y Subirà (2004), podría decirse que la mujer de Ca L'Estrada presenta una mayor estatura y robustez que otras mujeres neolíticas.

SF-502: Las tareas de remoción arqueológica pusieron en evidencia los restos de un segundo enterramiento, en este caso infantil. El individuo estaba representado exclusivamente por la extremidad superior izquierda, el fémur derecho, parte del tórax y del cráneo. Así pues, los índices de preservación eran muy bajos $\left(\mathrm{IP}_{1}=25 \%, \quad \mathrm{IP}_{2}=15.79 \%\right.$, $\mathrm{IP}_{3}=18.18 \%$ ) como consecuencia del trabajo de las máquinas en la excavación. A pesar de ello, el estado de conservación de los mismos era relativamente bueno, si tenemos en cuenta que en uno de los fragmentos de la mandíbula se preservan aún los alveólos desde el segundo incisivo derecho hasta el primer premolar izquierdo (figura 5).

Conserva in situ las piezas deciduales: 81 (primer incisivo inferior derecho), 71 (primer incisivo inferior izquierdo), 72 (segundo incisivo inferior izquierdo), 73 (canino inferior izquierdo) y 74 (primer premolar inferior izquierdo). Asimismo la propia fractura de la mandíbula permite ver la gran cavidad alveolar correspondiente a la corona definitiva del primer incisivo inferior derecho (41) y la corona de la pieza 34 (primer premolar inferior izquierdo). El estudio del desarrollo dental indica que se trata de un individuo infantil de 5-6 años de edad. Llama la atención el fuerte desgaste dental que presentan los dientes incisivos $(71,72$ y 81$)$ a pesar de su corta edad. En cuanto a la determinación del sexo, la conformación del fragmento de la mandíbula muestra un cuerpo alto y robusto, con un mentón bien desarrollado. Estos caracteres morfológicos indican que probablemente se está ante los restos de un individuo de sexo masculino (SCHUTKOWSKI, 1993).

\section{4.- INTERPRETACIÓN Y CONCLUSIONES}

El yacimientos de Ca l'Estrada presentaba una compleja y extensa estratigrafía que hacía muy difícil su excavación, en especial sobre aquellas estructuras arqueológicas que estaban situadas a gran profundidad. A ello se sumaba el condicionante temporal, intrínseco a una intervención de urgencia, que impidió abordar la excavación en extensión, horizontal y verticalmente. Por todo ello, la campaña arqueológica se limitó a la realización de un conjunto de sondeos.

Las estructuras funerarias documentadas en Ca l'Estrada, de las que se desconocen en gran parte cómo era su morfología completa, nos hablan posiblemente de fosas excavadas, de entre 40 y $80 \mathrm{~cm}$ de profundidad. Teniendo como referencia la Sepultura SF-501, el cuerpo del indivi-
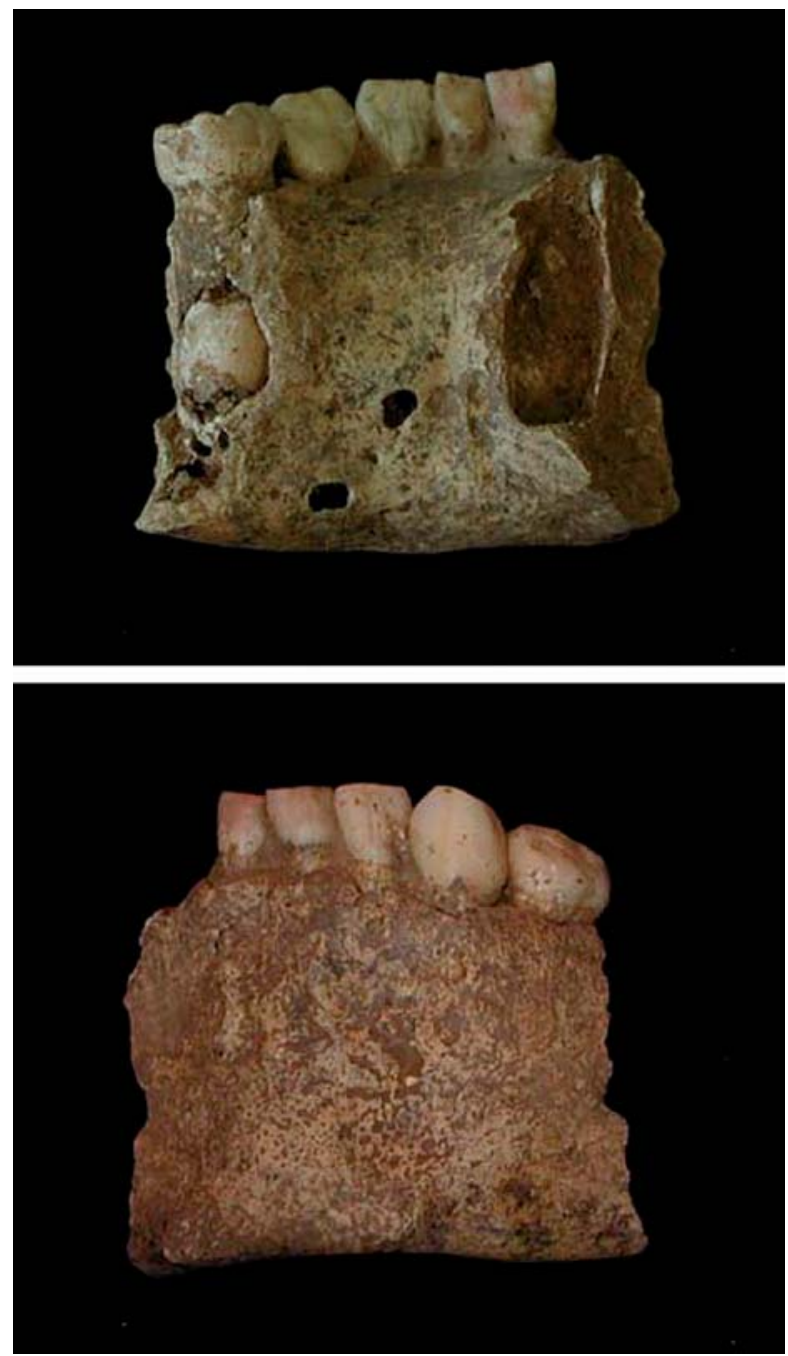

Fig. 5. Fotografia de la mandíbula SF-502. / SF-502 jaw.

duo femenino inhumado se encontraba en posición fetal en decúbito lateral, con las extremidades dobladas delante del tronco en una posición muy forzada. La posición es tan prieta que además de que debía hallarse envuelto en una mortaja, se baraja la posibilidad de que pudiera haber estado atado.

Es muy común en los contextos funerarios de este periodo, así como en los de momentos posteriores, la presencia de instrumentos líticos tallados. En sepulturas más o menos contemporáneas a Ca l'Estrada ha sido habitual encontrar como ajuar láminas de este tipo de sílex, que irán decreciendo en representatividad a medida que nos aproximamos a finales del V milenio. Será entonces cuando sean más habituales los instrumentos líticos tallados confeccionados en sílex melado. Los ejemplos más relevantes de láminas sílex como la de Ca l'Estrada se documentan en Sant Pau del Camp y Reina Amàlia, en la provincia de Barcelona, o d'Hort d'en Grimau y Pujolet de Moja, en el Penedès (MESTRES, 1988/1989; MESTRES et al., 1997; Gibaja, 2003; González et al., 2011; Borrell \& Gibaja, 2013). 


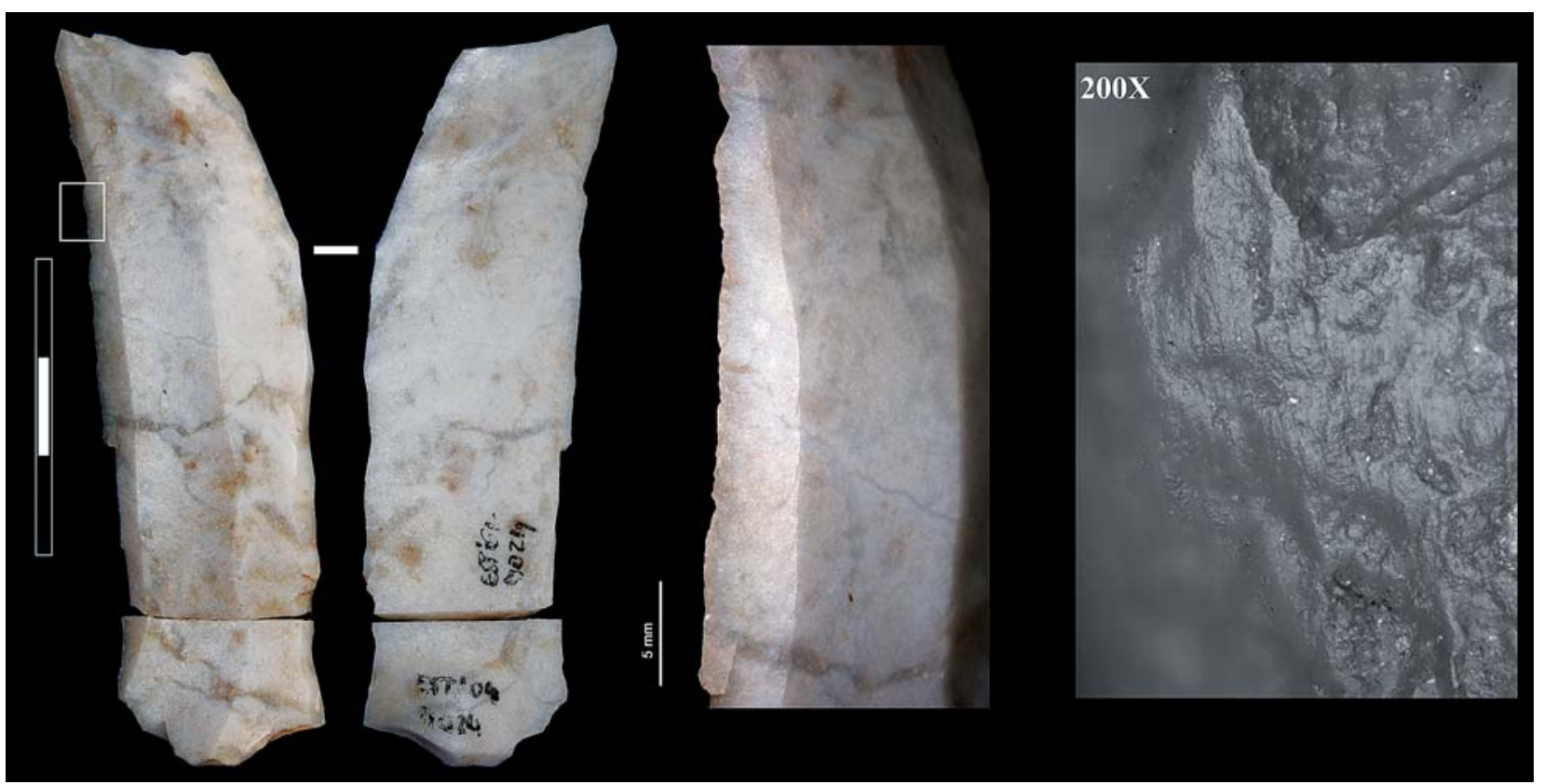

Fig. 6. Làmina de sílex localizada bajo la pelvis de la inhumación SF-501. / flint blade located under the pelvis of the SF-501 burial.

En definitiva, los enterramientos de Ca l'Estrada, a pesar de los inconvenientes metodológicos explicitados en relación a la excavación, son el reflejo de las primeras prácticas funerarias efectuadas por grupos neolíticos plenamente establecidos en el noreste peninsular. Desde mediados del $\mathrm{V}$ milenio cal BC, tales enterramientos constituyen algunos de los precedentes del tipo de comportamiento funerario que a finales de este mismo milenio se sistematizará en la conocida como "Cultura de los Sepulcros de Fosa". En los últimos años, descubrimientos como los de Ca l'Estrada han permitido retrasar los inicios de este tipo de enterramientos algunos siglos más atrás de lo que se suponía.

\section{BIBLIOGRAFÍA}

\section{ALFONSO, J., SUBIRÀ, M.E. \& MALGOSA, A.}

2000 Estudi antropològic de les inhumacions Neolítiques del Jaciment de Mas d'En Boixos (Pacs, Alt Penedès). Memòries d'intervencions arqueològiques a Catalunya. Departament de Cultura de la Generalitat de Catalunya. Inédito.

ALLIESE, F., ROIG, J., COLL, J.M., SUBIRÀ, M.E., RUIZ, J., CHAM$B O N, P H . \&$ GIBAJA, J.F.

2014 Les pratiques funéraires dans la nécropole du Néolithique moyen de Can Gambús-1 (Sabadell, Espagne): de la préparation du corps à la fermeture de la tombe. Bulletin de la Société préhistorique française 111, 453-468.

\section{BLASCO, A., EDO, M. \& VILLALBA, M.J.}

2005 Cardial, epicardial y postcardial en Can Sadurní (Begues, Baix Llobregat). El largo fin del Neolítico Antiguo en Cataluña, en Actas III Congreso del Neolítico en la Península Ibérica. 867-878. Santander.
BORRELL, F. \& GIBAJA, J.F.

2013 The First Neolithic Communities in North-East Iberia: procurement, production and use of lithic tools at the settlement of Caserna de Sant Pau del Camp (Barcelona, Spain) Journal of Island \& Coastal Archaeology 7, 313-337.

BOSCH, A. \& TARRÚS, J.

1991 La cova sepulcral del Neolític antic de l'Avellaner, Cogolls, Les Planes d'Hostoles (La Garrotxa). Girona, Sèrie Monogràfica 11, Centre d'Investigacions Arqueològiques. Girona.

\section{BOSCH GIMPERA, P.}

1913-14 Necròpolis a Sant Genís de Vilassar, en Secció arqueològica, excavacions i troballes. Anuari de I'Institut d'Estudis Catalans V, part II, 806-810.

BRAVO, P. HINOJO, E., SUBIRÀ, M.E., ALLIĖSE, F., MASCLANS, A., SANTANA, J., SANTOS, F.J., AGULLÓ, L., GÓMEZ-MARTÍNEZ, I., REMOLINS, G. \& GIBAJA, J.F.

2015 Les sépultures néolithiques de Can Gambús-2 (Sabadell, Espagne): une grande inconnue dans le contexte de la culture des Sepulcros de fosa. L'Anthropologie 119, 38-57.

BRONK, R

2010 http://c14.arch.ox.ac.uk/oxcal.html.

BROTHWELL, D.R.

1987 Desenterrando huesos. Ed. Fondo de Cultura Económica. México.

CAMPILLO, D. \& SUBIRÀ, M.E.

2004 Antropología Física para Arqueólogos. Ariel. Barcelona. 
CASTANY, J.

1992 Montboló i Chassey a Grioteres: Vilanova de Sau, Osona. Estratigrafia, paleoecologia, paleoeconomia i datació, en Estat de la Investigació sobre el Neolític a Catalunya. Actas 9è Col.loqui International d'Arqueologia de Puigcerdà. 150-152. Puigcerdà.

\section{CLEMENTE, I. \& GIBAJA, J.F.}

1998 Working processes on cereals: an approach through microwear analysis. Journal of Archaeological Science 25/5, 457-464.

\section{CRÉTOT, M.}

1978 L'arcade dentaire humaine. Morphologie. Julián Prélat. Paris.

\section{DUDAY, H., COURTAUD, P., CRUBEZY, E., SELLIER, P. \& TILLIER, A.M. \\ 1990 L'Anthropologie «de terrain»: reconnaissance et interpré- tation des gestes funéraires. Bulletins et mémoires de la Société d'anthropologie de Paris 2(3-4), 39-49.}

DUDAY, $\mathrm{H}$.

2009 The Archaeology of the Dead: Lectures in Archaeothanatology. Oxbow Books. Oxford.

FEREMBACH, D., SCHWIDETZKY, I. \& STLOUKAL, M.

1980 Recommendations for Age and Sex Diagnoses of Skeletons. Journal of Human Evolution 9, 517-549.

FERNÁNDEZ-LÓPEZ, J., SALAZAR-GARCÍA, D.C., SUBIRÀ, M.E., ROCA DE TOGORES, C., GÓMEZ-PUCHE, M., RICHARDS, M.P. \& ESQUEMBRE-BEBIA, M.A.

2012 Late Mesolithic burials at Casa Corona (Villena, Spain): direct radiocarbon and palaeodietary evidence of the last forager populations in Eastern Iberia. Journal of Archaeological Science 40, 671-680. doi: 10.1016/j.jas.2012.09.005.

\section{FORTÓ, A., MARTÍNEZ, P. \& MUÑOZ, V.}

2005 L'estàtua-menhir antropomorfa de Ca l'Estrada (Canovelles, Vallès Oriental). Cota Zero 20, 17-22.

2006 Ca l'Estrada (Canovelles, Vallès Oriental): un exemple d'ocupació de la plana vallesana des de la prehistòria a l'alta edat mitjana. Tribuna d'Arqueologia 2004/2005, 45-70.

2008 Las estructuras de combustión de grandes dimensiones de Ca l'Estrada en el neolítico europeo, en Actas IV Congreso del Neolítico en la Península Ibérica. 306-314. Alicante.

GIBAJA, J.F.

2003 Comunidades Neolíticas del Noreste de la Península Ibérica. Una aproximación socio-económica a partir del estudio de la función de los útiles líticos. BAR International Series S1140. Oxford.

GIBAJA, J.F., SUBIRÀ, M.E., TERRADAS, X., SANTOS, F.J., AGULLÓ, L., GOOMEZ-MARTÍNEZ, I., ALLIĖSE, F. \& FERNÁNDEZLÓPEZ DE PABLO, J.

2015 The Emergence of Mesolithic Cemeteries in SW Europe: Insights from the El Collado (Oliva, Valencia, Spain) radiocarbon record. PLOS ONE 10, 1-18. doi:10.1371/journal.pone.0115505
GONZÁLEZ, J., HARZBECHER, K. \& MOLIST, M.,

2011 Un nou assentament del V mil.leni a la costa de Barcelona. Quarhis 7, 86-100.

GRANADOS, O., PUIG, F. \& FARRÉ, R.

1993 La intervenció arqueològica a Sant Pau del Camp: un nou jaciment prehistòric al Pla de Barcelona. Tribuna d'Arqueologia 1991-1992, 27-32. Barcelona.

INIZIAN, M.L., REDURON, M., ROCHE, H. \& TIXIER, J.

1995 Technologie de la pierre taillée. CREP. Préhistoire de la pierre taillé 4. Meudon.

KROGMAN, W.M. \& ISCAN, M.Y.

1986 The Human Skeleton in Forensic Medicine. C.C. Thomas, Publ. Springfield.

LOVEJOY, C.O., MEINDL, R.S., PRYZBECK, T.R. \& MENSFORTH, R.P.

1985 Chronological metamorphosis of the auricular surface of the ilium: A new method for the determination of age at death. American Journal of Physical Anthropology 68, 15-28.

MARTIN, R. \& SALLER, K.

1957 Lehrbuch der Anthropologie. Ed. Gustav Fischer. Sttutgart.

MASSET, C

1982 Estimation de l'age au décés par les sutures craniennes. Tesis Doctoral. Université París VII. París.

MEINDL, R.S. \& LOVEJOY, C.O.

1985 Ectocraneal suture closure: A revised method for the determination of skeletal age at death and blind tests of its accuracy. American Journal of Physical Anthropology 68, 57-66.

MESTRES, J.

1988-89 Les sepultures neolítiques de l'Hort d'en Grimau (Castellví de la Marca, Alt Penedès). Olerdulae, Revista del Museu de Vilafranca 1-4, 97-129.

MESTRES, J., NADAL, J., SENABRE, M.R., SOCIAS, J. \& MORAGAS, N

1997 El Pujolet de Moja (Olèrdola, Alt Penedès), ocupació d'un territori durant el neolític i la primera edad del ferro. Tribuna d'Arqueologia 1995-1996, 121-148.

MOLIST, M., CRUELLS, W. \& CASTELLS, J.

1987 L'àrea megalítica de Tavertet (Osona). Cota Zero 3, 55-68.

MOLIST, M., VICENTE, O. \& FARRÉ, R.

2008 El jaciment de la caserna de Sant Pau del Camp: aproximació a la caracterització d'un assentament del neolític antic. Quaris 4, 14-24.

OLIVIER, G.

1960 Pratique Anthropologique. Vigot Frères. Paris. 
ORTÍ, F.

1990 Las formaciones evaporíticas del Terciario continental de la zona de contacto entre la Cuenca del Ebro y los Catalánides, (Ortí, F. \& Salvany, M., eds.), en ORTÍ, F. \& SALVANY, M. (Eds.) Formaciones evaporíticas de la Cuenca del Ebro y cadenas periféricas, y de la zona de Levante. ENRESA. 133-154. Universidad de Barcelona. Barcelona.

PALOMO, A.

2012 Tecnologia lítica i de la fusta de la Prehistoria recent al nordest peninsular. Anàlisi tecnomorfològica i experimental. Tesis doctoral. Universidad Autónoma de Barcelona.

POU, R., MARTÍ, M., JORDANA, X., MALGOSA, A.\& GIBAJA, J.F.

2010 L'enterrament del neolític antic de la Plaça de la Vila de Madrid (Barcelona). Una estructura funeraria del Vlè mil.leni aC. Quaris 6, 94-107.

ROSELL, J., LINARES, R.\& LLOMPART, C.

2001 El "Garumniense" Prepirenaico. Revista de la Sociedad Española de Geología 14(1-2), 47-56.

RUIZ, J. \& SUBIRÀ, M.E.

2004 Informe antropològic de l'estructura 16 del jaciment de Els Cirerers (Neolític antic) (Vilafranca del Penedès). Memòries d'intervencions arqueològiques a Catalunya. Departament de Cultura de la Generalitat de Catalunya. Inédito.

\section{SAFONT, S., ALESAN, A. \& MALGOSA, A.}

1999 Memòria de l'excavació realitzada a la tomba del carrer Nou, 12 (Sant Bartomeu del Grau, Osona). Memòries d'intervencions arqueològiques a Catalunya. Departament de Cultura de la Generalitat de Catalunya. Inédito.

\section{SCHUTKOWSKI, $\mathrm{H}$.}

1993 Sex determination of infant and juvenile skeletons: 1. morphognostic features. American Journal of physical Anthropology $90,199-205$.
SUBIRÀ, M.E.

2004 Estudi de les restes antropològiques procedents de Ca l'Estrada (Canovelles, Vallès Oriental), en MARTÍNEZ, P. FORTÓ, A. \& MUÑOZ, V. Memòria científica de les intervencions arqueològiques a Ca l'Estrada, Servei d'Arqueologia de la Generalitat de Catalunya. 2007. Memòries d'intervencions arqueològiques a Catalunya. Departament de Cultura de la Generalitat de Catalunya. Inédito.

SUBIRÀ, M.E., GARCIA, E. \& BERROCAL, I.

2004 Informe de les restes humanes recuperades a l'estructura 11 d'Horts de Can Torras (Castellar del Vallès, Vallès Occidental). Memòries d'intervencions arqueològiques a Catalunya. Departament de Cultura de la Generalitat de Catalunya. Inédito.

TEN, $R$

1980 Notes entorn del neolític vallesà. Arraona 10, 6-25.

TERRADAS, X., ORTEGA, D. \& BOIX, J.

2012 El projecte LitoCAT. Creació d'una litoteca de referència de roques silícies de Catalunya. Tribuna d'Arqueologia 2010-2011, 131-150.

UBELAKER, D.H.

1989 Human Skeletal Remains. Smithsonian Institution. Washington.

VILLALBA, M.J., BLASCO, A. \& EDO, M.

2004 Memòria d'excavacions realitzades a la cova de Can Sadurní. Campanya 2002. Memòries d'intervencions arqueològiques a Catalunya. Departament de Cultura de la Generalitat de Catalunya. Inédito.

2006 Memòria d'excavacions realitzades a la cova de Can Sadurní. Campanya 2004. Memòries d'intervencions arqueològiques a Catalunya. Departament de Cultura de la Generalitat de Catalunya. Inédito.

WALKER, P.L., JOHNSIN, J.R. \& LAMBERT, P.M.

1988 Age and Sex Biases in the Preservation of Human Skeletal Remains. American Journal of Physical Anthropology 76, 183-188. 
